\title{
The ever-expanding immunomodulatory role of calreticulin in cancer immunity
}

\author{
Marco de Bruyn ${ }^{1+}$, Valerie R. Wiersma ${ }^{2 \dagger}$, Wijnand Helfrich ${ }^{2}$, Paul Eggleton ${ }^{3}$ and Edwin Bremer ${ }^{2,3} *$ \\ ${ }^{1}$ Department of Gynecologic Oncology, University Medical Center Groningen (UMCG), University of Groningen, Groningen, Netherlands \\ ${ }^{2}$ Department of Surgery, Translational Surgical Oncology, University Medical Center Groningen (UMCG), University of Groningen, Groningen, Netherlands \\ ${ }^{3}$ University of Exeter Medical School, Exeter, UK
}

\section{Edited by:}

Marek Michalak, University of

Alberta, Canada

Reviewed by:

Stephan Gasser, National University

of Singapore, Singapore

Mohey Eldin El Shikh, Queen Mary

University of London, UK

Antonella Sistigu, Istituto Superiore di

Sanità, Italy

*Correspondence:

Edwin Bremer, Department of

Surgery, Translational Surgical

Oncology, University Medical Center

Groningen, University of Groningen,

Hanzeplein 1, BA44, Groningen 9713

GZ, Netherlands

e-mail:e.bremer@umcg.nl

${ }^{\dagger}$ Marco de Bruyn and Valerie

R. Wiersma have contributed equally

to this work.
Calreticulin is a pleiotropic molecule that normally resides in the lumen of the endoplasmic reticulum (ER). Here, it has various functions, ranging from regulation of calcium homeostasis to ensuring proper protein folding. More recently, calreticulin gained special interest for its extracellular functions, where it has direct immunomodulatory activity. In this respect, calreticulin activates dendritic cells and macrophages. In addition, certain anti-cancer therapies induce the translocation of calreticulin from the ER to the cell surface of dying cancer cells, where calreticulin dictates the immunogenicity of these cells. Interestingly, treatment with tumor necrosis factor (TNF)-related apoptosis inducing ligand (TRAIL) also induces membrane calreticulin exposure on cancer cells. As shown here, calreticulin directly interacts with TRAIL and its receptor-signaling complex, as well as with other TNF family members. Of note, TRAIL is a well known immunomodulatory molecule, and is expressed on the surface of natural killer T-cells. Therefore, calreticulin may have an as yet unrecognized wide(r) impact on immunity, with the TNF-ligand family modulating virtually all aspects of the immune response.

Keywords: calreticulin, TNF, tumor necrosis factor related apoptosis inducing ligand, immunomodulation, immunogenic cell death, complex formation

\section{INTRODUCTION}

The endoplasmic reticulum (ER)-resident protein calreticulin is a pleiotropic molecule with many functions in the ER, ranging from protein folding, calcium homeostasis, and regulation of loading of antigenic peptides into major histocompatibility class I (MHCI) $(1,2)$. Thus, ER-resident calreticulin has an important impact on development and correct execution of immunity. However, in recent years, calreticulin has taken center stage not for its ER-related functions, but for its newly uncovered immunomodulatory effects in the extracellular space. It has become evident that ER chaperones such as calreticulin function as danger associated molecular pattern molecules (DAMPs) once outside the cell (3). For instance, when present on the cell surface of dendritic cells (DCs) calreticulin functions as a receptor for autocrineproduced complement factor $1 \mathrm{q}(\mathrm{Clq})$, which is upregulated during CD40L/CD40 signaling, a key $\mathrm{T}$ helper signal required for effective DC maturation (4). In addition, soluble calreticulin promotes differentiation of $\mathrm{CD} 1 \mathrm{dhi} \mathrm{CD}^{+}{ }^{+} \mathrm{B}$-cells into antibodysecreting cells (5). Further, soluble calreticulin induced TNF- $\alpha$ and IL-6 release from macrophages, which was regulated by scavenger receptor A (6).

A further prominent pro-immunogenic feature of extracellular calreticulin is its key role in the process of immunogenic cell death (ICD) (7). ICD is a type of apoptosis that is induced by certain chemotherapeutics or radiation, which promotes phagocytic uptake of apoptotic cells by professional antigen presenting cells, i.e., myeloid-derived DCs. Briefly, anthracycline or radiation therapy trigger the translocation of calreticulin to the pre-apoptotic tumor cell surface, which is dependent on the induction of an ER-stress response $(8,9)$. Cell surface exposed calreticulin then promotes clearance of the dying cancer cells by DCs, with subsequent release of ATP, HMGB1, and HSP70 from late apoptotic cells giving requisite cues for DC maturation and clonal T-cell expansion (10).

Immunogenic cell death has been described in a preclinical setting for an ever-expanding set of cytotoxic therapies, including many chemotherapeutics, radiation therapy, and photodynamic therapy. Interestingly, also treatment of cancer cells with a recombinant form of the tumor necrosis factor (TNF)-related apoptosis inducing ligand (TRAIL) was reported to induce calreticulin exposure and ICD (11). TRAIL is a member of the TNF superfamily that can bind to a set of five receptors; TRAILR1 (DR4), TRAILR2 (DR5), TRAIL-R3 (DcR1, TRID), TRAIL-R4 (DcR2, TRUNDD), and soluble osteoprotegerin. Binding of TRAIL to its agonistic receptors TRAILR1 and TRAILR2 induces assembly of the so-called death inducing signaling complex (DISC) to the intracellular death domain (DD). The DISC contains the adaptor molecule Fas-associated protein with death domain (FADD) and the pro-form of initiator caspase-8, which is auto-proteolytically processed in the DISC. Activation of caspase- 8 triggers a proteolytic caspase cascade that ultimately leads to the execution of apoptosis. Of note, the pro-apoptotic signaling of the DISC can be inhibited by recruitment of additional regulators, such as cFLIP (12). 
TNF-related apoptosis inducing ligand is an important immune effector molecule that is expressed on various types of immune cells including natural killer (NK) cells, T-cells, and natural killer T-cells (NKT cells) (13). Expression of TRAIL on T-cells is further increased during T-cell receptor (TCR) stimulation in the presence of interferon- $\gamma(\operatorname{IFN} \gamma)$ (14). Similarly, IFN $\gamma$ mediated activation of NK-cells, monocytes, and DCs enhances the expression of TRAIL on these cells $(15,16)$. TRAIL and its receptors (TRAILRs) play an important role in anti-tumor immunity, e.g., being involved in the immune surveillance for (metastatic) cancer cells by liver NK-cells (17). Thus, the induction of calreticulin exposure upon treatment with recombinant TRAIL may be related to a possible immunoregulatory effect of TRAIL in the context of its normal role on immune effector cells. Interestingly, calreticulin can also interact directly with TRAIL in a catching-type ELISA, as well as with the immunoregulatory TNF family members such as CD40 ligand (CD40L) and FasL (18). In contrast, calreticulin did not interact with TNF- $\alpha$, adiponectin, or CD30L. The reported activation of ICD upon treatment with recombinant TRAIL suggests that calreticulin may regulate the pro-immunogenic effect of TRAIL during anti-tumor immunity.

\section{CALRETICULIN DIRECTLY INTERACTS WITH PRO-APOPTOTIC TNF-LIGANDS AND RECEPTOR COMPLEXES}

Calreticulin was recently reported to bind to TNF family member FasL in the synovial fluid of rheumatoid arthritis (RA) patients. This FasL/calreticulin interaction inhibited FasL-induced apoptosis of Jurkat T-cells (19). Since patients with RA have elevated levels of calreticulin in their serum, this might inhibit apoptosis of inflammatory T-cells in this particular disease. Our own studies confirm a similar direct interaction of calreticulin with TRAIL, as evidenced by, e.g., fluorescent microscopy for calreticulin and TRAIL on the cell surface of TRAIL-treated A375M melanoma cells and not in control cells (Figure 1A). This is in line with the reported translocation of calreticulin to the cell surface of apoptotic HeLa cells by recombinant TRAIL (11). Interestingly, calreticulin also strongly co-localized with TRAILR2 on TRAILtreated A375M cells (Figures 1B,C), with calreticulin, TRAIL, and TRAILR2 all being in one complex co-precipitated upon anti-HATRAIL immunoprecipitation experiments (Figure 1D). Similarly, calreticulin was immunoprecipitated in the FasL/Fas DISC (data not shown). The association of calreticulin with TRAILR2 occurs in a clear patched pattern on the cell surface (Figures 1B,C), a pattern corresponding to that of the patched foci to which calreticulin redistributed on the cell membrane of apoptotic neutrophils (20), on mitoxantrone treated CT26 colon cancer cells (21), and hypericin-treated bladder cancer cells (22).

The recruitment of calreticulin in distinct patches on the cell membrane has been shown to facilitate engulfment of apoptotic cells by phagocytes induced by UV radiation or anthracycline therapy $(7,20)$ (Figure 2A). These calreticulin-rich patches also contained high levels of phosphatidylserine (PS), an apoptotic marker that provides strong "eat me" signals for phagocytes (20). Correspondingly, calreticulin binds in a $\mathrm{Ca}^{2+}$-dependent manner directly to the polar head of PS with high affinity $\left(K_{\mathrm{D}}=1.5 \times 10^{-5} \mathrm{M}\right)(24)$. This recruitment of calreticulin into lipid rafts was dependent on ERp57 $(21,25)$. Of note, on the surface of viable cells calreticulin is associated with CD47, a prominent so-called "don't eat me" signal that blocks phagocytosis by binding to the inhibitory receptor SIRP $\alpha$ on phagocytes (26). During apoptosis, calreticulin dissociates from CD47 and associates with PS; a shift that promotes phagocytosis (20). Of note, the balance between pro-phagocytic calreticulin and anti-phagocytic CD47 has also been described in multiple human cancer cells, with cancer cells often being characterized by CD47 overexpression to elude phagocytic removal $(26,27)$. Similarly, our experiments indicate that calreticulin dissociates from CD47 upon TRAIL treatment of A375M cells (Figure 1C). Together with literature, this dissociation suggests that the interaction of calreticulin with TRAIL and TRAILR2 may facilitate phagocytic clearance (Figure 2B).

\section{CALRETICULIN IS RECRUITED TO ACTIVE SIGNALING COMPLEXES OF THE TNFR SUPERFAMILY}

In an earlier study on TNFR-signaling, surface expressed calreticulin was reported to be recruited to TNF receptor 1 (TNFR1) and its adaptor TNFR-associated death domain (TRADD) upon treatment with bacterial peptidoglycan $N$-acetylmuramyl-L-alanyl-Disoglutamine ( $\mathrm{L}, \mathrm{D}-\mathrm{MDP}$ ) (28). This interaction was independent of TNF and triggered apoptosis in $\mathrm{RK}_{13}$ kidney cells that could be blocked by anti-calreticulin antibodies. Similarly, calreticulin associated with TRAIL, TRAILR2, as well as adaptor protein FADD and pro-caspase- 8 in A375M melanoma cells (Figures 1D,E). Of note, caspase- 8 was predominantly present in its pro-form after 15 min of TRAIL treatment, but was in its processed p $41 / 43$ form after $180 \mathrm{~min}$. Further, caspase- 8 processing occurred mainly at $37^{\circ} \mathrm{C}$, although treatment on ice also induced DISC formation (Figures 1D,E). The interaction of calreticulin with TRAIL was necessary for its association with TRAILR2 as calreticulin was not co-immunoprecipitated with TRAILR2 upon treatment with agonistic TRAILR2 antibody, despite DISC formation (Figure 1F). Thus, physical interaction of TRAIL with calreticulin mediates its recruitment to the TRAILR2 DISC.

\section{CALRETICULIN ASSOCIATION WITH THE TRAILR2-DISC DEPENDS ON RAFT REORGANIZATION BUT IS INDEPENDENT OF APOPTOTIC SIGNALING}

During ICD with mitoxantrone, the maximum level of surface exposed calreticulin was observed within $15 \mathrm{~min}$, and remained at stable levels for at least $24 \mathrm{~h}$ (7). In line with this, surface calreticulin became visible after $30 \mathrm{~min}$ on neutrophils and continued to increase as neutrophils became more apoptotic (20). In contrast, induction of ICD upon treatment of Hela cells with TRAIL only induced detectable levels of calreticulin on the surface after $24 \mathrm{~h}$ of incubation (11). On A375M cells, calreticulin translocated to and associated with calreticulin with TRAIL already after $15 \mathrm{~min}$, with maximal association after $1 \mathrm{~h}$ (Figure 1G), which corresponded to the maximum level of processed caspase-8 (p43/41) (Figure 1H). Further, the maximum association of calreticulin with TRAIL and the DISC occurs before the execution of apoptosis, as determined by activation of effector caspases-3 and -7 (Figure $\mathbf{1 H}$ ). This dynamic corresponds to the pre-apoptotic exposure of calreticulin during ICD (7). Interestingly, induction of apoptosis was required to induce cell surface calreticulin exposure upon treatment with mitoxantrone, with depletion of caspase- 8 completely abolishing 

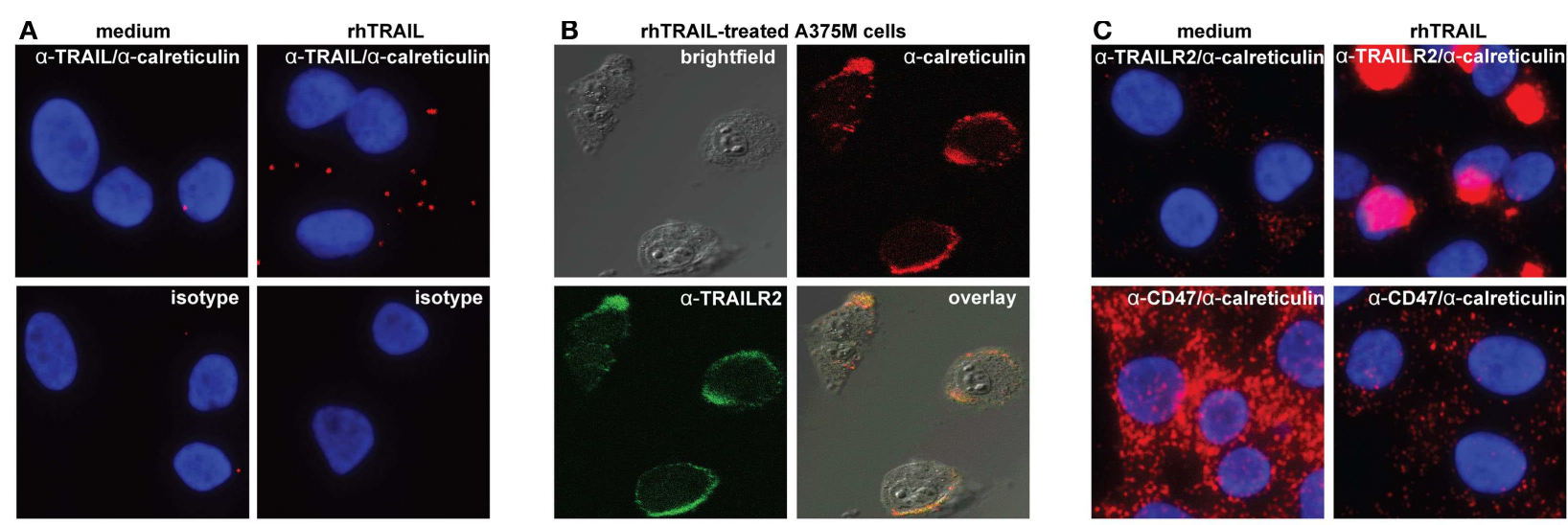

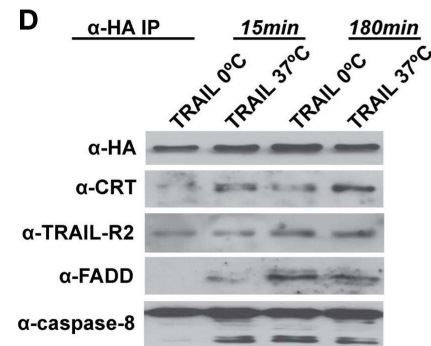

H

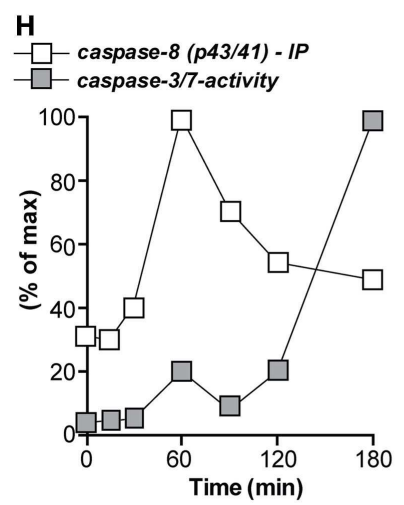

E $\frac{\alpha \text {-calreticulin IP }}{180 \min }$

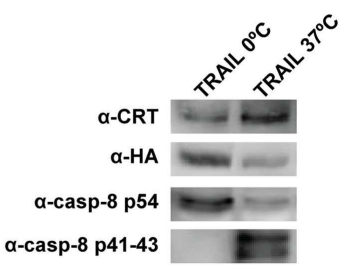

I

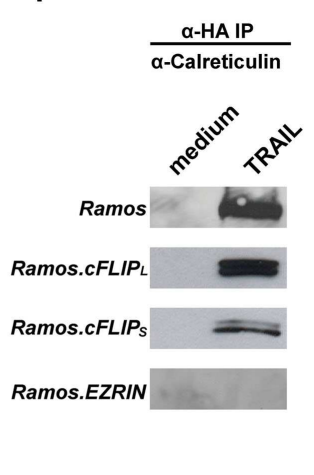

$\mathbf{F}$ $\frac{\text { Q-TRAIL-R2 IP }}{60 \min }$
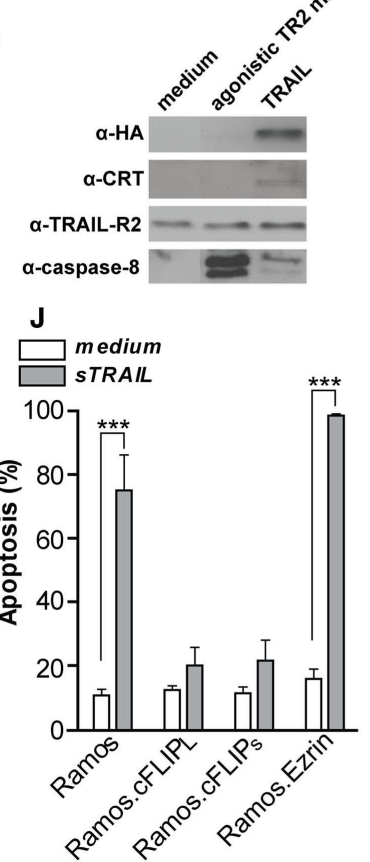

G

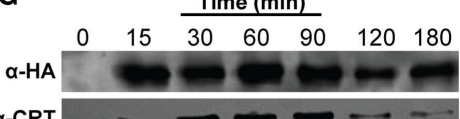

$\alpha-C R T+\cdots$

\section{Time (min)}

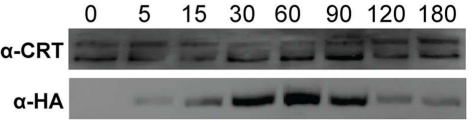

K

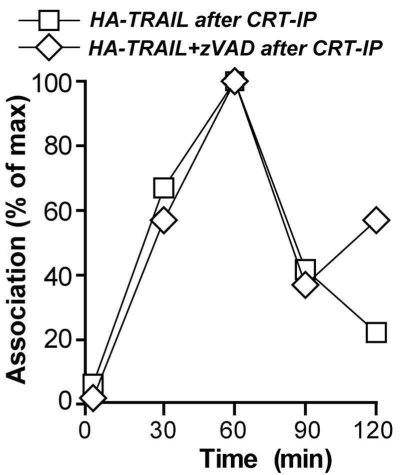

FIGURE 1 | Calreticulin binds to TRAIL and associates with the

TRAILR2-DISC. (A) A375M cells were treated with $100 \mathrm{ng} / \mathrm{ml}$ rhTRAIL for $1 \mathrm{~h}$, incubated with mAbs directed against calreticulin (Ab2907, Abcam) and TRAIL (B-S23, Diaclone), or isotype controls, and subsequently analyzed by proximity ligation assay (Duolink II, Olink Biosciensce) (distance between both proteins; $<40 \mathrm{nM}$ or $\sim 4-12$ proteins). (B) A375M cells were treated with $100 \mathrm{ng} / \mathrm{ml}$ rhTRAIL for $1 \mathrm{~h}$, double stained for calreticulin (red; Ab2907, Abcam) and TRAILR2 (green; HS-201, Alexis) and analyzed using confocal fluorescent microscopy. (C) As in (A), but the proximity ligation assay were performed using mAbs directed against calreticulin (Ab2907, Abcam), TRAILR2 (HS-201, Alexis), and CD47 (ab3283, Abcam). (D) A375M cells were treated with HA-TRAIL [constructed by cloning the CDNA of HA-tagged TRAIL in frame into previously described vector pEE14 (23), yielding pEE14-HA-TRAIL] for 15 or $180 \mathrm{~min}$ at the indicated temperatures. Cells were subsequently lysed and HA-TRAIL was precipitated via affinity tag purification (HA-sepharose beads; 3F10, Roche). Precipitates were subsequently probed for the presence of HA (3F10, Roche), calreticulin (Ab39818, Abcam), TRAILR2 (HS-201, Alexis), FADD (272S, cell signaling), and caspase-8 (18C8, cell signaling). (E) As in (D), but precipitated using anti-calreticulin. Precipitates were subsequently probed for the presence of calreticulin, $\mathrm{HA}$, and caspase-8. (F) A375M melanoma cells were treated with HA-TRAIL or agonistic TRAILR2 $\mathrm{mAb}$ for $60 \mathrm{~min}$ at $37^{\circ} \mathrm{C}$. Cells were subsequently lysed andTRAILR2 was precipitated. The precipitates were probed for the presence of HA, calreticulin, TRAILR2, and caspase-8. (G) A375M cells were treated with HA-TRAIL at $37^{\circ} \mathrm{C}$ for the indicated time-points. Cells were subsequently lysed and HA-TRAIL (upper panel) or calreticulin (lower panel) were precipitated. Precipitates were subsequently probed for the presence of calreticulin or HA-TRAIL, respectively. (H) A375M cells were treated with HA-TRAIL at $37^{\circ} \mathrm{C}$ for the indicated time-points. $\square$ Quantification of caspase-8 (p42/41) association with TRAIL as determined by immunoprecipitation — caspase-3/-7 activity as determined by flow cytometry (CellEvent caspase-3/-7 probe, Invitrogen). (I) Ramos, Ramos.cFLIP , Ramos.cFLIP , and Ramos. Ezrin cells were treated with HA-TRAIL for $1 \mathrm{~h}$ at $37^{\circ} \mathrm{C}$. Cells were subsequently lysed and HA-TRAIL was precipitated via affinity tag purification. Precipitates were subsequently probed for the presence of calreticulin. (J) Ramos, Ramos.cFLIP , Ramos.cFLIP , and Ramos.Ezrin cells were treated with rhTRAIL for $16 \mathrm{~h}$ and apoptosis was assessed (DioC6 staining, flow cytometry using BD Accuri Flow cytometer). *** $p<0.001$. (K) A375M cells were treated with HA-TRAIL in the presence or absence of $20 \mu \mathrm{M}$ zVAD-fmk (CalBiochem). Subsequently, calreticulin was precipitated and the precipitates were stained for HA-TRAIL. Association of calreticulin and TRAIL is depicted as percentage of maximum association. 


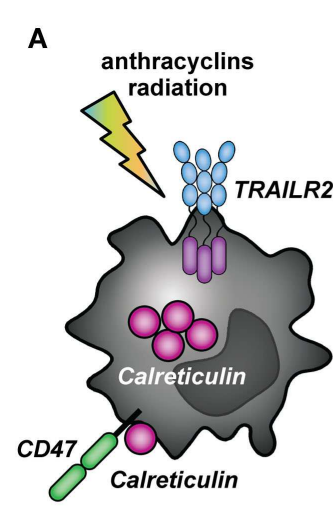

tumor cell

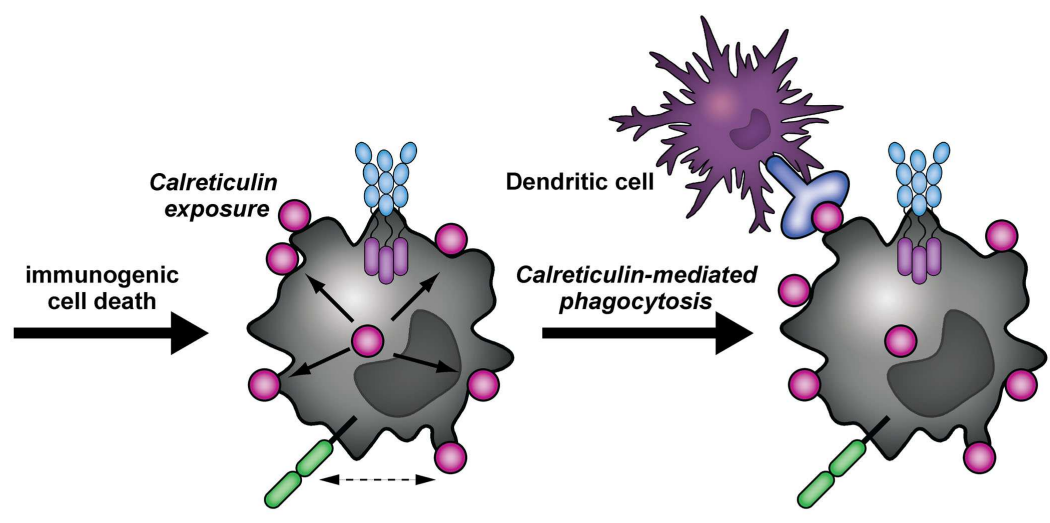

pre-apoptotic tumor cell

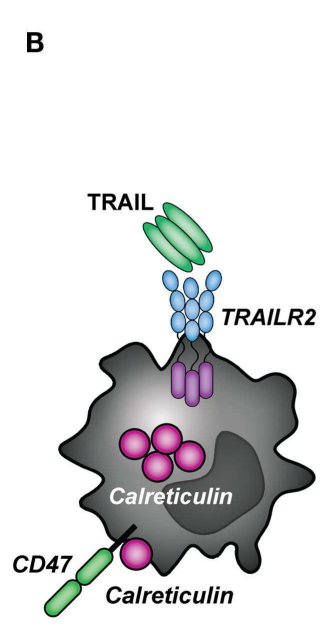

tumor cell

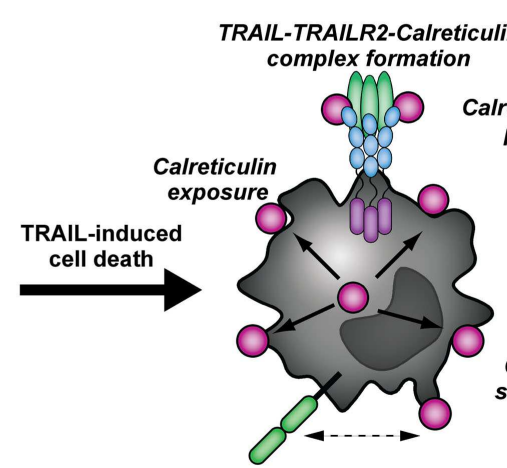

pre-apoptotic tumor cell
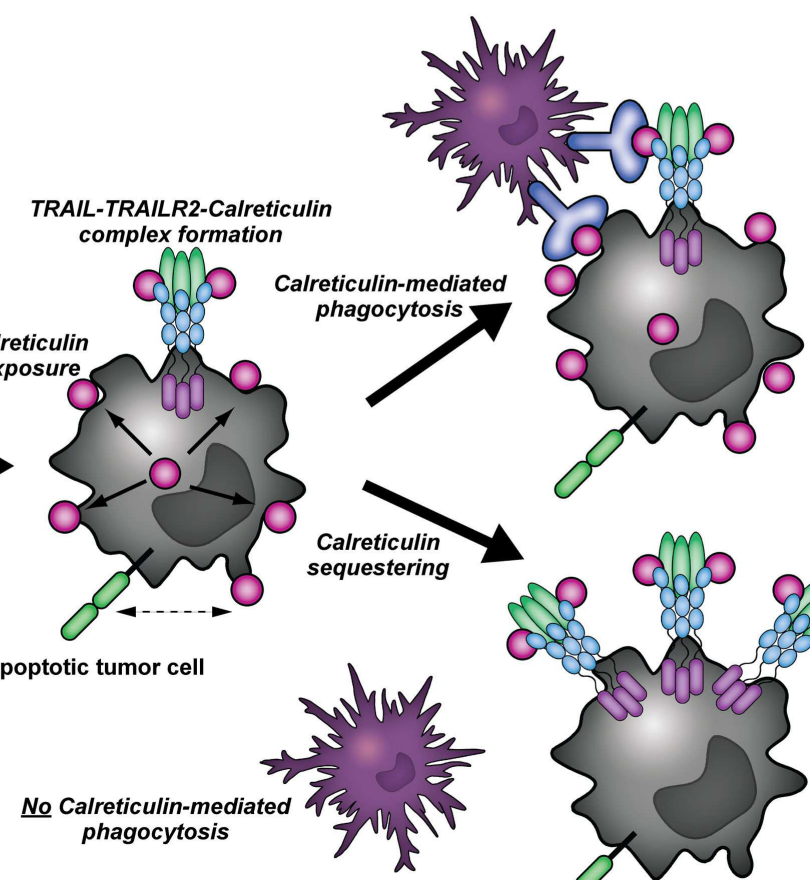

Calreticulin equestering

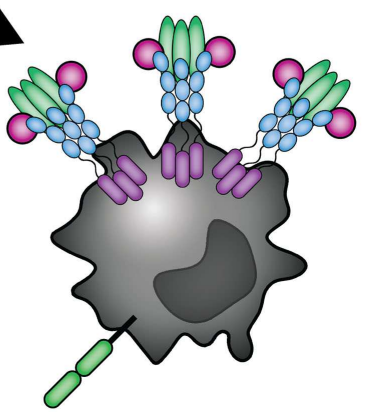

FIGURE 2 | Immunomodulatory role of calreticulin in cancer immunity (A). In (tumor) cells, calreticulin predominantly resides in the ER. Upon induction of immunogenic cell death (ICD) by, for instance, anthracycline or radiation therapy, calreticulin translocates to the surface of pre-apoptotic cells. In addition, surface exposed calreticulin (eat me signal) dissociates from CD47 (don't eat me signal), whereupon the apoptotic cancer cells can be recognized and taken up by DCs. (B) The binding of TRAIL to its receptor (TRAILR2) results in the translocation of calreticulin to the cell surface of cancer cells, whereby a complex is formed between TRAIL, TRAILR2-DISC, and calreticulin. Simultaneously, calreticulin dissociates from CD47. The formation of the TRAIL/TRAILR2/ Calreticulin-complex may have different outcomes: (1) As described for the concept of ICD, cell surface exposure of calreticulin and dissociation from CD47 induced by TRAIL treatment may facilitate phagocytic uptake by DCs. (2) the binding of calreticulin to TRAIL and TRAILR2 may impair phagocytic clearance by DCs, as calreticulin may be segregated away from the membrane microdomains in which it can partake in the phagocytic uptake by DCs. the translocation of calreticulin (11). In contrast, inhibition of caspase-8 did not inhibit calreticulin exposure upon photodynamic therapy with hypericin (9). In line with the latter report, calreticulin was still co-precipitated with TRAIL in Ramos B-cells that are resistant to TRAIL-mediated apoptosis by overexpression of the anti-apoptotic DISC component cFLIP (Ramos.cFLIP L $_{\mathrm{L}}$ and Ramos.cFLIP ) (Figures 1I,J). Correspondingly, co-treatment of A375M cells with TRAIL and pan-caspase-inhibitor zVADfmk did not abrogate the association of calreticulin with TRAIL (Figure 1K).

During ICD, the actin cytoskeleton was required for translocation of calreticulin to the pre-apoptotic cell surface (11). Similarly, calreticulin-TRAIL association was dependent on cytoskeletal rearrangements and was abolished in Ramos cell overexpressing 
the double negative Ezrin mutant (DN-Ezrin), which is incapable of interacting with actin (Figure 1I). Thus, calreticulin association with the DISC is independent of apoptosis, but dependent on cytoskeletal reorganization.

\section{CONCLUSION/FUTURE DIRECTIONS}

It is clear from literature that treatment of cancer cells with TRAIL can induce calreticulin relocalization to the cell surface. Further, as evidenced by the data in the current manuscript, surface calreticulin is recruited to the TRAIL/TRAILR/DISC complex and dissociates from CD47. Together, these observations suggest that calreticulin could promote phagocytosis of apoptotic cells upon TRAIL-mediated killing (Figure 2B). On the other hand, its association with the TRAILR2-DISC might render calreticulin unable to effectuate pro-immunogenic removal of target cells by DCs. In this respect, it is tempting to speculate that by binding to the TRAILR2-DISC, calreticulin is segregated away from the membrane microdomains in which it can partake in the phagocytic uptake of target cells by DCs or other phagocytes (Figure 2B). These findings would be consistent with the non-immunogenic clearance of cells undergoing TRAIL-mediated programed cell death (29). To gain insight in the possible immunomodulatory role of calreticulin during TRAIL-induced apoptosis, it will be imperative to characterize the particular complex or membrane microdomain in which calreticulin resides in the context of ICD. Comparison of calreticulin localization upon treatment with typical immunogenic inducers, and how this compares to its localization during TRAIL-mediated cell death may shed light on its particular role.

The inhibition of FasL-mediated T-cell death by calreticulin, the direct induction of apoptosis by L,D-MDP-bound calreticulin via TNFR1 and the here described association of calreticulin to TRAILR2 during TRAIL-mediated cell death suggest a much broader role for calreticulin in the antitumor immune response than as a mediator of ICD. By directly modulating apoptosis and associating with key apoptotic players, calreticulin could function as a bridge between innate and adaptive immunity. In this paradigm, induction of programed cell death by immune effector molecules, such as TRAIL and FasL, induces a rapid pre-apoptotic translocation of calreticulin to the cell surface. On the cell surface, calreticulin can then either modulate induction of cell death as described for FasL (19) or translocate to specific cell surface microdomains (with or without receptors of the TNF superfamily), thereby modulating phagocytosis and pro-immunogenic DC-mediated removal. In its soluble state, secretion of calreticulin in response to apoptotic stimuli can directly affect the immediate early innate immune response by modulating cytokine secretion of macrophages. Interestingly, agonistic TRAIL-receptors have been reported as negative regulators of innate immune responses (30). An intriguing possibility currently being investigated in our laboratory is that sequestration of calreticulin by TRAIL-receptors could contribute to limiting activation of innate immunity.

Calreticulin exposure can further trigger activation of the adaptive immune response by promoting immunogenic DC-uptake of apoptotic cells during ICD. In this respect, it is also of interest that of all the TNF ligands it was found to interact with in a catching-type ELISA, calreticulin most strongly associated with CD40L. Thus, recruitment of calreticulin to CD40L/CD40 signaling complexes may impact DC activity. Whether calreticulin directly associates with TNF family members beyond CD40, FasL, and TRAIL has not been identified, to date. However, the reported interaction of calreticulin with several TNF-ligand family members points to the possibility that calreticulin may have an as yet unrecognized wide(r) impact on immunity, with the TNF ligand family modulating virtually all aspects of the immune response.

\section{ACKNOWLEDGMENTS}

This work was supported by Dutch Cancer Society grants RUG 2009-4355 (EB), RUG2009-4542, RUG2011-5206, RUG20125541, RUG2013-6209 (to EB/WH), the Netherlands Organization for Scientific Research (EB), and the European Communities Seventh Framework Programme (FP7/2007-2013) under grant agreement 215009 RedCat (PE).

\section{REFERENCES}

1. Michalak M, Milner RE, Burns K, Opas M. Calreticulin. Biochem J (1992) 285:681-92.

2. Eggleton P, Michalak M. Calreticulin for better or for worse, in sickness and in health, until death do us part. Cell Calcium (2013) 54:126-31. doi:10.1016/j. ceca.2013.05.006

3. Krysko DV, Garg AD, Kaczmarek A, Krysko O, Agostinis P, Vandenabeele P. Immunogenic cell death and DAMPs in cancer therapy. Nat Rev Cancer (2012) 12:860-75. doi: $10.1038 / \mathrm{nrc} 3380$

4. Baruah P, Dumitriu IE, Malik TH, Cook HT, Dyson J, Scott D, et al. C1q enhances IFN-gamma production by antigen-specific $\mathrm{T}$ cells via the CD40 costimulatory pathway on dendritic cells. Blood (2009) 113:3485-93. doi:10.1182/blood-200806-164392

5. Zhang T, Xia Y, Zhang L, Bao W, Hong C, Gao XM. CD1d(hi)CD5(+) B cells differentiate into antibody-secreting cells under the stimulation with calreticulin fragment. Protein Cell (2013) 4:872-81. doi:10.1007/s13238-013-3062-5

6. Duo CC, Gong FY, He XY, Li YM, Wang J, Zhang JP, et al. Soluble calreticulin induces tumor necrosis factor-alpha (TNF-alpha) and interleukin (IL)-6 production by macrophages through mitogen-activated protein kinase (MAPK) and NFkappaB signaling pathways. Int J Mol Sci (2014) 15:2916-28. doi:10.3390/ijms15022916

7. Obeid M, Tesniere A, Ghiringhelli F, Fimia GM, Apetoh L, Perfettini JL, et al. Calreticulin exposure dictates the immunogenicity of cancer cell death. Nat Med (2007) 13:54-61. doi:10.1038/nm1523

8. Martins I, Kepp O, Schlemmer F, Adjemian S, Tailler M, Shen S, et al. Restoration of the immunogenicity of cisplatin-induced cancer cell death by endoplasmic reticulum stress. Oncogene (2011) 30:1147-58. doi:10.1038/onc.2010.500

9. Garg AD, Krysko DV, Verfaillie T, Kaczmarek A, Ferreira GB, Marysael T, et al. A novel pathway combining calreticulin exposure and ATP secretion in immunogenic cancer cell death. EMBO J (2012) 31:1062-79. doi:10.1038/ emboj.2011.497

10. Apetoh L, Ghiringhelli F, Tesniere A, Obeid M, Ortiz C, Criollo A, et al. Toll-like receptor 4-dependent contribution of the immune system to anticancer chemotherapy and radiotherapy. Nat Med (2007) 13:1050-9. doi:10. $1038 / \mathrm{nm} 1622$

11. Panaretakis T, Kepp O, Brockmeier U, Tesniere A, Bjorklund AC, Chapman DC, et al. Mechanisms of pre-apoptotic calreticulin exposure in immunogenic cell death. EMBO J (2009) 28:578-90. doi:10.1038/emboj.2009.1

12. Bremer $E$. Targeting of the tumor necrosis factor receptor superfamily for cancer immunotherapy. ISRN Oncol (2013) 2013:371854. doi:10.1155/2013/371854

13. Falschlehner C, Schaefer U, Walczak H. Following TRAIL's path in the immune system. Immunology (2009) 127:145-54. doi:10.1111/j.1365-2567.2009.03058.x

14. Kayagaki N, Yamaguchi N, Nakayama M, Eto H, Okumura K, Yagita H. Type I interferons (IFNs) regulate tumor necrosis factor-related apoptosisinducing ligand (TRAIL) expression on human T cells: a novel mechanism for the antitumor effects of type I IFNs. J Exp Med (1999) 189:1451-60. doi:10.1084/jem.189.9.1451 
15. Griffith TS, Wiley SR, Kubin MZ, Sedger LM, Maliszewski CR, Fanger NA. Monocyte-mediated tumoricidal activity via the tumor necrosis factor-related cytokine, TRAIL. J Exp Med (1999) 189:1343-54. doi:10.1084/jem.189.8.1343

16. Fanger NA, Maliszewski CR, Schooley K, Griffith TS. Human dendritic cells mediate cellular apoptosis via tumor necrosis factor-related apoptosis-inducing ligand (TRAIL). J Exp Med (1999) 190:1155-64. doi:10.1084/jem.190.8.1155

17. Cretney E, Takeda K, Yagita H, Glaccum M, Peschon JJ, Smyth MJ. Increased susceptibility to tumor initiation and metastasis in TNF-related apoptosis-inducing ligand-deficient mice. J Immunol (2002) 168:1356-61. doi:10.4049/jimmunol. 168.3.1356

18. Duus K, Pagh RT, Holmskov U, Hojrup P, Skov S, Houen G. Interaction of calreticulin with CD40 ligand, TRAIL and Fas ligand. Scand J Immunol (2007) 66:501-7. doi:10.1111/j.1365-3083.2007.01999.x

19. Tarr JM, Winyard PG, Ryan B, Harries LW, Haigh R, Viner N, et al. Extracellular calreticulin is present in the joints of patients with rheumatoid arthritis and inhibits FasL (CD95L)-mediated apoptosis of T cells. Arthritis Rheum (2010) 62:2919-29. doi:10.1002/art.27602

20. Gardai SJ, McPhillips KA, Frasch SC, Janssen WJ, Starefeldt A, Murphy-Ullrich JE, et al. Cell-surface calreticulin initiates clearance of viable or apoptotic cells through trans-activation of LRP on the phagocyte. Cell (2005) 123:321-34. doi:10.1016/j.cell.2005.08.032

21. Panaretakis T, Joza N, Modjtahedi N, Tesniere A, Vitale I, Durchschlag M, et al The co-translocation of ERp57 and calreticulin determines the immunogenicity of cell death. Cell Death Differ (2008) 15:1499-509. doi:10.1038/cdd.2008.67

22. Garg AD, Krysko DV, Vandenabeele P, Agostinis P. Hypericin-based photodynamic therapy induces surface exposure of damage-associated molecular patterns like HSP70 and calreticulin. Cancer Immunol Immunother (2012) 61:215-21. doi:10.1007/s00262-011-1184-2

23. de Bruyn M, Rybczynska AA, Wei Y, Schwenkert M, Fey GH, Dierckx RA, et al. Melanoma-associated chondroitin sulfate proteoglycan (MCSP)-targeted delivery of soluble TRAIL potently inhibits melanoma outgrowth in vitro and in vivo. Mol Cancer (2010) 9:301. doi:10.1186/1476-4598-9-301

24. Paidassi H, Tacnet-Delorme P, Verneret M, Gaboriaud C, Houen G, Duus $\mathrm{K}$, et al. Investigations on the $\mathrm{Clq}$-calreticulin-phosphatidylserine interactions yield new insights into apoptotic cell recognition. J Mol Biol (2011) 408:277-90. doi:10.1016/j.jmb.2011.02.029
25. Obeid M. ERP57 membrane translocation dictates the immunogenicity of tumor cell death by controlling the membrane translocation of calreticulin. J Immunol (2008) 181:2533-43. doi:10.4049/jimmunol.181.4.2533

26. Chao MP, Jaiswal S, Weissman-Tsukamoto R, Alizadeh AA, Gentles AJ, Volkmer J, et al. Calreticulin is the dominant pro-phagocytic signal on multiple human cancers and is counterbalanced by CD47. Sci Transl Med (2010) 2:63ra94. doi:10.1126/scitranslmed.3001375

27. Jaiswal S, Jamieson CH, Pang WW, Park CY, Chao MP, Majeti R, et al. CD47 is upregulated on circulating hematopoietic stem cells and leukemia cells to avoid phagocytosis. Cell (2009) 138:271-85. doi:10.1016/j.cell.2009.05.046

28. Chen D, Texada DE, Duggan C, Liang C, Reden TB, Kooragayala LM, et al. Surface calreticulin mediates muramyl dipeptide-induced apoptosis in RK13 cells. J Biol Chem (2005) 280:22425-36. doi:10.1074/jbc.M413380200

29. Green DR, Ferguson T, Zitvogel L, Kroemer G. Immunogenic and tolerogenic cell death. Nat Rev Immunol (2009) 9:353-63. doi:10.1038/nri2545

30. Diehl GE, Yue HH, Hsieh K, Kuang AA, Ho M, Morici LA, et al. TRAIL-R as a negative regulator of innate immune cell responses. Immunity (2004) 21:877-89. doi:10.1016/j.immuni.2004.11.008

Conflict of Interest Statement: The authors declare that the research was conducted in the absence of any commercial or financial relationships that could be construed as a potential conflict of interest.

Received: 11 December 2014; accepted: 31 January 2015; published online: 20 February 2015.

Citation: de Bruyn M, Wiersma VR, Helfrich W, Eggleton P and Bremer E (2015) The ever-expanding immunomodulatory role of calreticulin in cancer immunity. Front. Oncol. 5:35. doi: 10.3389/fonc.2015.00035

This article was submitted to Tumor Immunity, a section of the journal Frontiers in Oncology.

Copyright (c) 2015 de Bruyn, Wiersma, Helfrich, Eggleton and Bremer. This is an openaccess article distributed under the terms of the Creative Commons Attribution License (CC BY). The use, distribution or reproduction in other forums is permitted, provided the original author(s) or licensor are credited and that the original publication in this journal is cited, in accordance with accepted academic practice. No use, distribution or reproduction is permitted which does not comply with these terms. 